\title{
Performance Evaluation of IEEE 1609 WAVE for Vehicular Communications
}

\author{
Jyh-Horng Wen ${ }^{1}$ and Chien-Erh Weng ${ }^{2}$ \\ ${ }^{1}$ Department of Electrical Engineering, Tunghai University, 40704 Taichung, Taiwan \\ ${ }^{2}$ Department of Electronic Communication Engineering, National Kaohsiung Marine University, 81157 Kaohsiung, Taiwan
}

Correspondence should be addressed to Chien-Erh Weng; ceweng@mail.nkmu.edu.tw

Received 27 December 2012; Revised 17 March 2013; Accepted 31 March 2013

Academic Editor: Cheng-Min Lin

Copyright ( 2013 J.-H. Wen and C.-E. Weng. This is an open access article distributed under the Creative Commons Attribution License, which permits unrestricted use, distribution, and reproduction in any medium, provided the original work is properly cited.

In IEEE 1609, it uses IEEE 802.11 Enhanced Distributed Channel Access (EDCA) mechanism to access the channel. IEEE 802.11 EDCA is a new wireless technology for wireless access in the vehicular environment (WAVE). It defines a new supplement to the existing IEEE 802.11 MAC protocol. In IEEE 802.11 EDCA, the aim is providing a QoS support. While the system serves different access categories (ACs), EDCA does not perform well under high load conditions. In order to improve the efficiency, we pay attention to the EDCA with transmit opportunity (TXOP) mechanism. We first proposed a Markov chain model and studied the behavior. We extend the model to support IEEE 802.11 EDCA and presented a more accurate analysis under nonideal channel environment. We also compared it with that without TXOP mechanism under channel error environment.

\section{Introduction}

In recent years, the WLANs market is experiencing an explosive growth. The medium access control (MAC) protocol is the key element that provides the efficiency in accessing the channel, while satisfying the quality of service (QoS) requirements. IEEE 802.11 EDCA is a new wireless technology which is an enhanced version of IEEE 802.11 distributed coordination function (DCF). The IEEE 802.11 EDCA aims at improving the capabilities and efficiency of the IEEE 802.11 MAC protocol by defining a new mechanism to support the QoS services. While the system serves different ACs, EDCA does not perform well under high load conditions. In order to improve the efficiency, EDCA provides two mechanisms named as transmission opportunity (TXOP) and Block_ACK. These two mechanisms are allowed to offer new data transmission services that include the multiple frame delivery $[1-3]$. There have been many performance analyses of the IEEE 802.11 EDCA. Deng and Chang [4] proposed a priority scheme by differentiating the backoff window. Aad and Castelluccia [5] proposed a priority scheme by differentiating interframe spaces (IFSs), in which a higher priority class uses IFS, whereas a lower priority class uses a space that equals the sum of IFS and the maximum window size. In [6] Veres et al. proposed priority schemes by differentiating the minimum backoff window size and the maximum window size. In [7], it studied the performance of the IEEE 1609 WAVE and IEEE 802.11p trial standards for vehicular communications. It had implemented key components of these standards in a simulation environment also supporting realistic vehicular mobility simulation. Gallardo et al. analyze the performance of EDCA under the specific conditions of the $\mathrm{CCH}$ of a WAVE environment. The protocol is modeled using Markov chains, and results related to throughput, frame-error rate, buffer occupancy, and delay are obtained under different traffic-load conditions [8]. In [9], adaptive Enhanced Distributed Channel Access (EDCA) mechanism based on IEEE 802.11p WAVE for vehicular ad hoc network (VANET) to improve medium access ratio is proposed. These performance studies neglected that high load situation. They cannot truly reflect the real operation of EDCA with priority schemes. In this paper, we pay attention to the EDCA of IEEE 802.11 with TXOP mechanism. We proposed a Markov chain model and studied the behavior. We 
extend the model to support IEEE 802.11 EDCA and present a more accurate analysis of the IEEE 802.11 EDCA under a nonideal channel environment in WAVE. We also compared it with that without TXOP mechanism under channel error environment.

The rest of this paper is organized as follows. In Section 2, a general description of IEEE 802.11 and our proposed model with TXOP mechanism is presented. Analytical performance deviations of proposed model including throughput analysis under non-ideal channel scenario are presented in Section 3. The numerical results are given with discussion in Section 4. Finally, conclusions are drawn in Section 5.

\section{Review of IEEE 802.11}

The IEEE 802.11 wireless local area network is a sharedmedium communication network that transmits information over wireless links for all IEEE 802.11 stations in its transmission range to receive. It is one of the most deployed wireless networks in the world and is likely to play a major role in multimedia home networks and next-generation wireless communications. IEEE 802.11 wireless networks can be configured into two different modes: ad hoc and infrastructure. In ad hoc mode, all wireless stations within the communication range can communicate directly with each other, whereas, in the infrastructure mode, an access point (AP) is needed to connect all stations to a distribution system (DS), and each station can communicate with others through the AP. IEEE 802.11 is composed of both a physical layer (PHY) and MAC specifications for wireless local area networks [10, 11].

In the IEEE802.11 protocol, the fundamental mechanism to access the medium is called the distributed coordination function (DCF). This is a random access scheme which is based on the Carrier Sense Multiple Access with Collision Avoidance (CSMA/CA) protocol. The standard also defines an optional point coordination function (PCF) which is based on a polled-response mechanism.

In the DCF, if a station has a frame to transmit, it will monitor the channel. If the channel is busy, the MAC waits until the medium becomes idle and then defers for an extra time interval, called the DCF interframe space (DIFS). After sensing the channel within a DIFS, the STA randomly chooses a backoff interval before transmitting. The backoff counter is decremented in terms of a time slot as long as the channel is sensed as being idle. The counter is stopped when a transmission with other STAs is detected on the channel and reactivated when the channel is sensed as being idle again for more than a DIFS. The station transmits its frame when the backoff counter reaches zero. At each transmission, the backoff time is uniformly chosen in the range $[0, W-1]$, where $W$ is the current backoff window size. $W$ equals the initial backoff window size $C W_{\min }$. After each unsuccessful transmission, $W$ is doubled until a maximum backoff window size value $C W_{\max }$ is reached. Once it reaches $C W_{\max }, W$ shall remain at the value $C W_{\max }$ until it is reset. $W$ shall be reset to $C W_{\min }$ after every successful transmission or the retransmission counter reaches the retry limit $(L)$.
After the destination station successfully receives the frame, it transmits an acknowledgment frame (ACK) following a short inter-frame space (SIFS) time. If the transmitting station does not receive the ACK within a specified ACK timeout or it detects the transmission of a different frame on the channel, it reschedules the frame transmission according to the previous backoff rules. The above mechanism is called the basic access mechanism (ACK CSMA/CA). To reduce the hidden station problem, an optional four-way handshakes data transmission mechanism called request-to-send (RTS)/clear-to-send (CTS) is also defined in the DCF. Before transmitting a packet, a station operating in an RTS/CTS mode "reserves" the channel by sending a special request-to-send short frame. The destination station acknowledges the receipt of a RTS frame by sending back a clear-to-send frame. Since collision may occur only on the RTS frame and it is detected by the lack of a CTS response, the RTS/CTS mechanism allows for an increase in the system performance by reducing the duration of a collision when long messages are transmitted [10-12].

2.1. Enhanced DCF of IEEE 802.11. IEEE 802.11 EDCA is a new wireless technology which is an enhanced version of IEEE 802.11 DCF. It defines a new supplement to the existing IEEE 802.11 MAC protocol. The IEEE 802.11 EDCA aims at improving the capabilities and efficiency of the IEEE 802.11 MAC protocol by defining a new mechanism to support the QoS services. EDCA specifies four default access categories (ACs). Each STA contends for the channel access and independently starts its backoff depending on its associated AC. Each AC uses AIFS [AC], $C W_{\text {min }}[\mathrm{AC}]$, and $C W_{\max }[\mathrm{AC}]$ instead of DIFS, $C W_{\min }$, and $C W_{\max }$ of the DCF [8].

The contention method of EDCA is the same as that in DCF. Each STA having a frame to transmit has to wait for the channel to be idle without interruption for a period AIFS [AC], and then it should start a random backoff process with its own $C W[\mathrm{AC}]$. For each time slot interval, during which the channel stays idle, the random backoff value is decremented. When the backoff counter reaches zero, the frame is transmitted. AIFS [AC] is calculated as follows:

$$
\text { AIFS }[\mathrm{AC}]=\text { AIFSN }[\mathrm{AC}] \times \text { a Slot Time }+ \text { a SIFS Time, }
$$

where the backoff time is calculated as follows:

$$
\text { backoff time }=\text { random_integer } \times \text { a Slot Time, }
$$

where random_integer is uniformly and randomly chosen in the range $[1, C W(\mathrm{AC})+1]$, instead of $[0, C W]$ as in the DCF. Initially, $C W$ of each $\mathrm{AC}$ is equal to $C W_{\min }[\mathrm{AC}]$. After each collision, $C W$ is doubled up to

$$
C W_{\max }[\mathrm{AC}]=2^{m} *\left(C W_{\min }[\mathrm{AC}]+1\right)-1,
$$

where $m$ is called the maximum backoff stage. Once it reaches $C W_{\max }[\mathrm{AC}]$, it remains at this value until it is reset.

2.2. The System Model. We assume in the following that, for a given station in the priority $i$ class, $b(i, t)$ is defined as a 


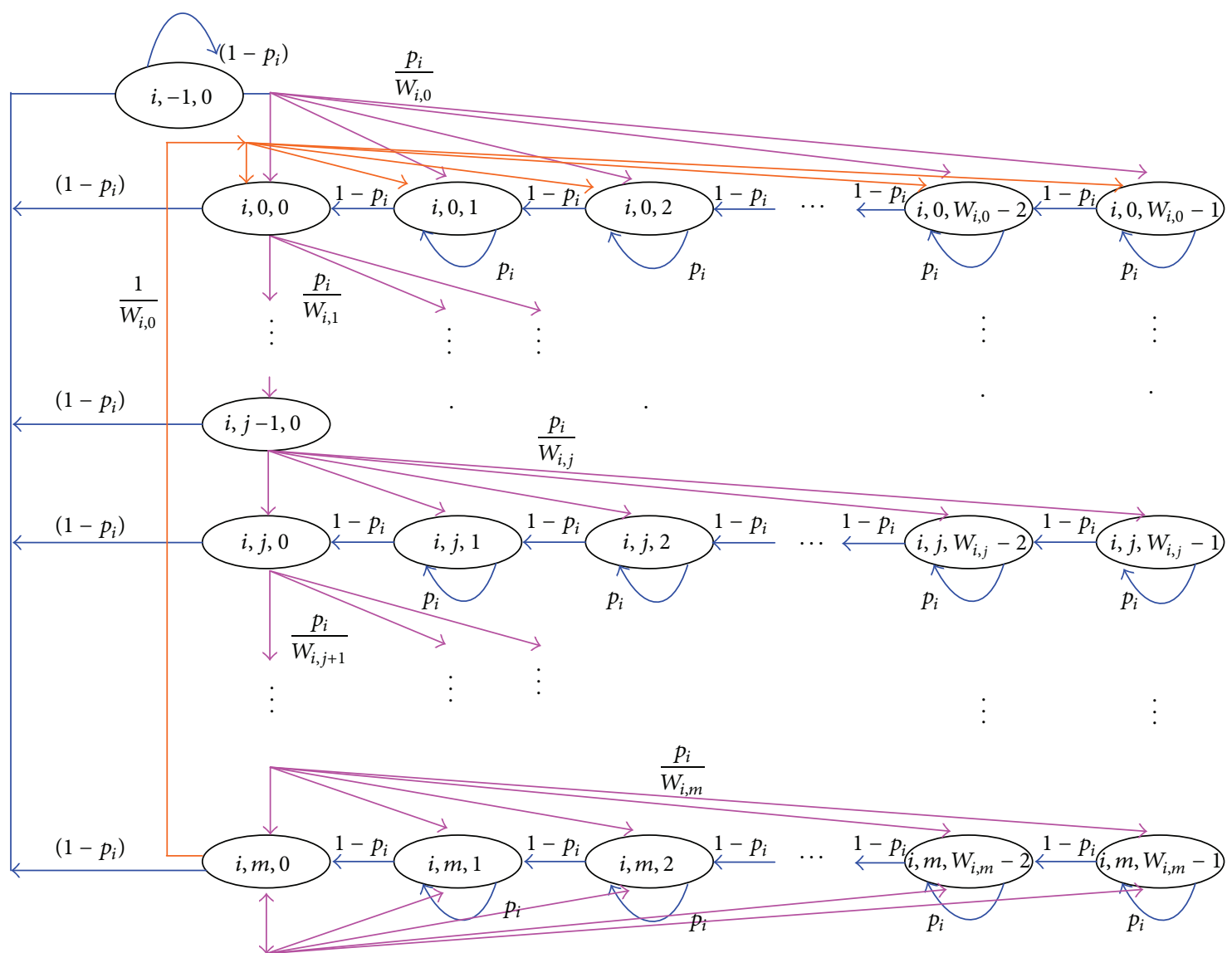

FIGURE 1: Markov chain model for the priority $i$ class with the backoff window size.

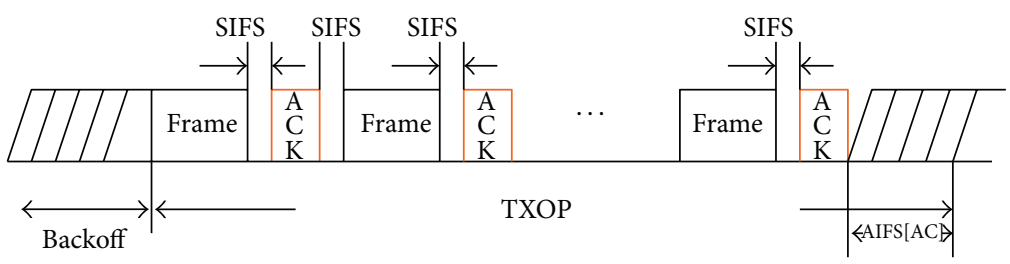

FiguRE 2: TXOP frame structure.

random process representing the value of backoff counter at time $t$, and $s(i, t)$ is defined as the random process representing the backoff stage $j$, where $0 \leq j \leq m$ and $m$ is the maximum backoff stage. The value of the backoff counter is uniformly chosen in the range $\left(0,1, \ldots, W_{i, j}-1\right)$, where $W_{i, j}=2^{j} W_{i, 0}$ and $W_{i, 0}=C W_{\min }[i]$. Let $p_{i}$ denote the probability that a transmitted frame collides with the priority $i$ class. A Markov chain $\{s(i, t), b(i, t)\}$ can be established to analyze the contention process. Therefore, the state of each station in the priority $i$ class is described by $\{i, j, k\}$, where $i$ is just an index standing for the priority $i$ class, $j$ stands for the backoff stage and takes values $(0,1, \ldots, m)$, and $k$ stands for the backoff delay and takes values $\left(0,1, \ldots, W_{i, j}-1\right)$ in time slots. The state transition diagram for the priority $i$ class is shown in Figure 1, where the state $\{i, 1,0\}$ stands for the state that the station senses when the channel is idle, and when a previous frame transmits successfully, the STA can transmit a frame immediately without activating the backoff stage. The transmission probabilities are listed as follows.

The backoff counter freezes when the STA senses that the channel is busy in the priority $i$ class:

$$
P\{i, j, k \mid i, j, k\}=p_{i}, \quad 0 \leq k \leq W_{i, j}-1,0 \leq j \leq m .
$$

The backoff counter decrements when the STA senses the channel is idle in the priority $i$ class:

$$
P\{i, j, k \mid i, j, k+1\}=1-p_{i}, \quad 0 \leq k \leq W_{i, j}-2,0 \leq j \leq m .
$$

The STA enters the $\{i,-1,0\}$ state if it has a successful transmission with the previous frame in the priority $i$ class:

$$
P\{i,-1,0 \mid i, j, 0\}=1-p_{i}, \quad 0 \leq j \leq m .
$$




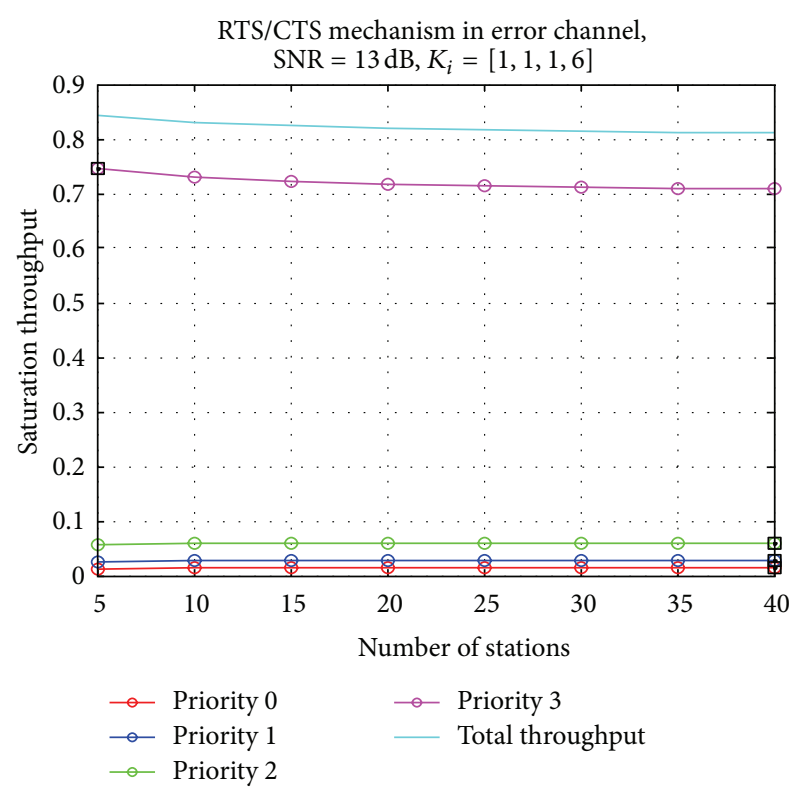

FIGURE 3: Saturation throughput of the different priority STAs with the TXOP mechanism under a channel error scenario, $K_{i}=$ $[1,1,1,6]$.

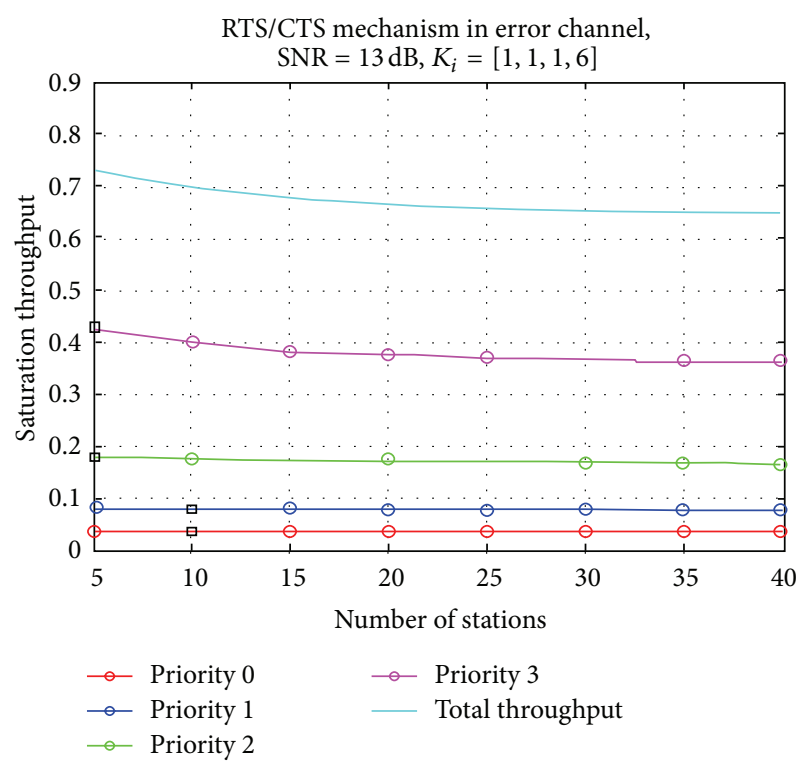

FIGURE 4: Saturation throughput of the different priority STAs without the TXOP mechanism under a channel error scenario, $K_{i}=$ $[1,1,1,6]$.

The STA transmits its frame without entering the backoff process at $\{i, 1,0\}$ state if it detects that its previous transmitted frame was successfully received and the channel is idle in the priority $i$ class:

$$
P\{i,-1,0 \mid i,-1,0\}=1-p_{i}
$$

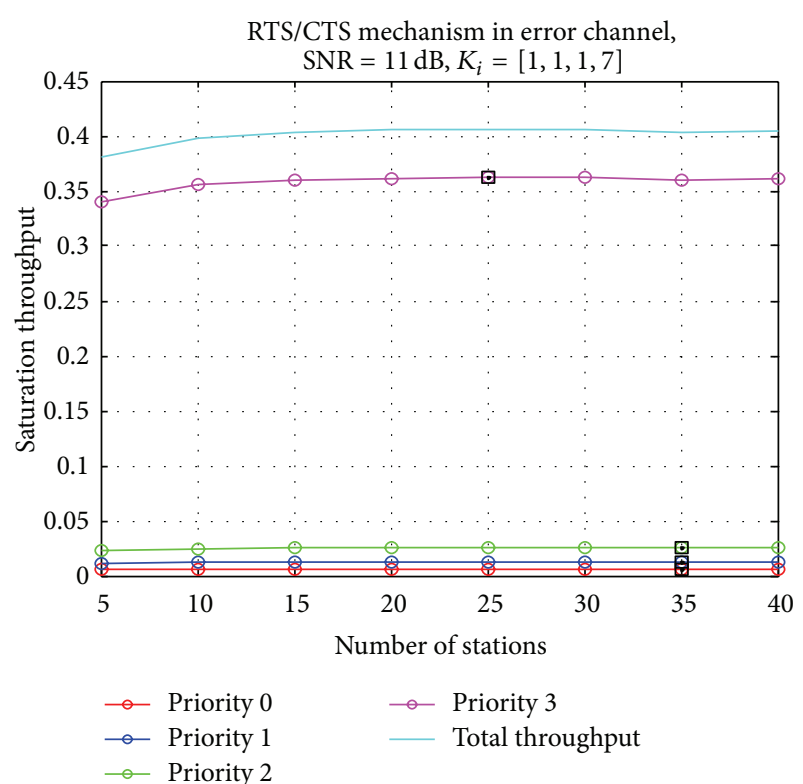

FIGURE 5: Saturation throughput of the different priority STAs with the TXOP mechanism under a channel error scenario, $K_{i}=$ $[1,1,1,7]$, and $\mathrm{SNR}=11 \mathrm{~dB}$.

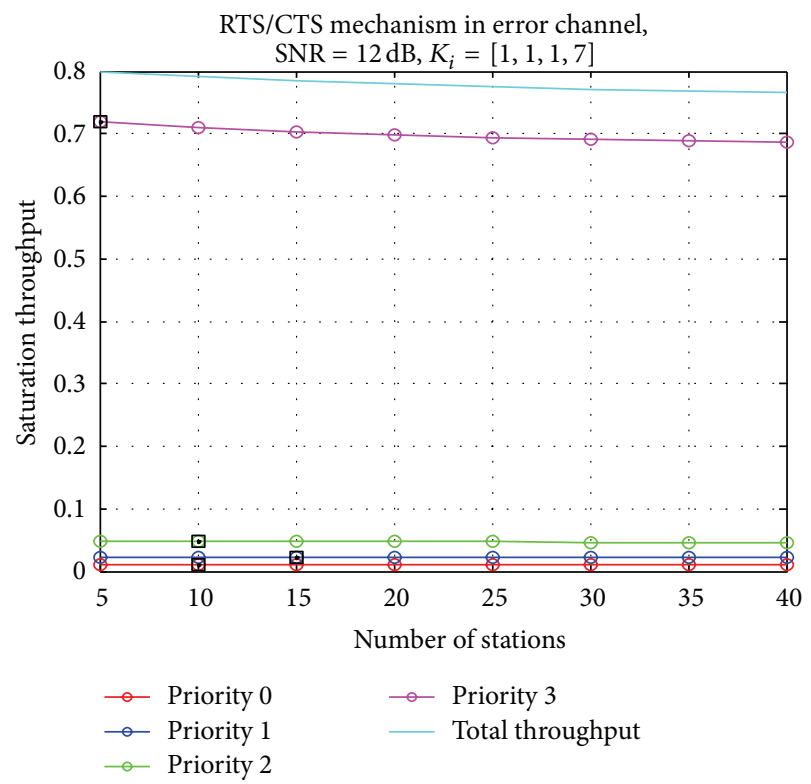

FIGURE 6: Saturation throughput of the different priority STAs with the TXOP mechanism under a channel error scenario, $K_{i}=$ $[1,1,1,7]$, and $\mathrm{SNR}=12 \mathrm{~dB}$.

The STA defers the transmission of a new frame and enters stage 0 of the backoff process if the STA finds a collision has occurred at $\{i, 1,0\}$ state in the priority $i$ class:

$$
P\{i, 0, k \mid i,-1,0\}=\frac{p_{i}}{W_{i, 0}}, \quad 0 \leq k \leq W_{i, 0}-1 .
$$




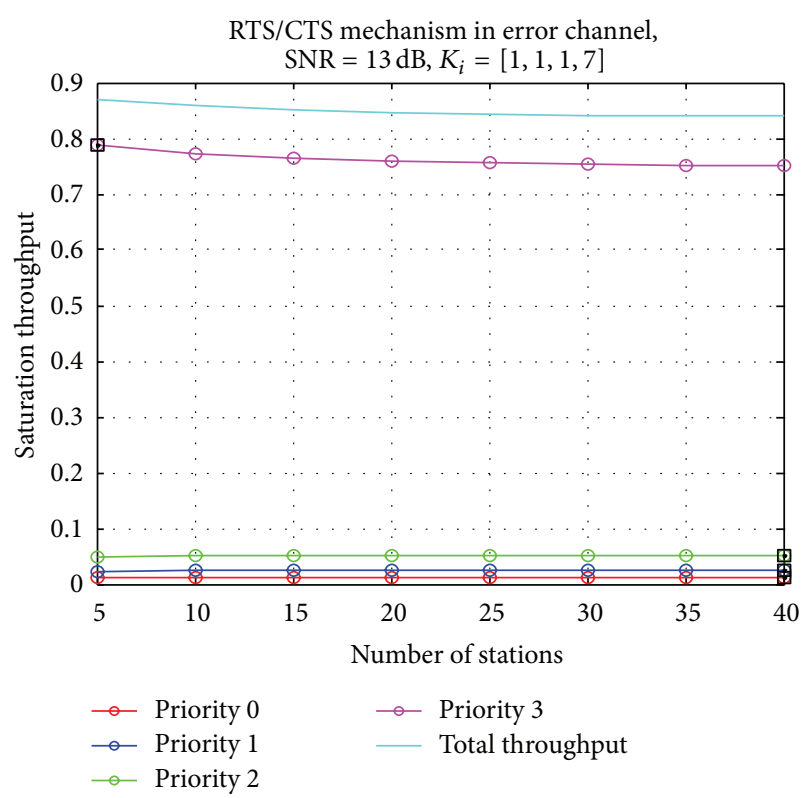

FIGURE 7: Saturation throughput of the different priority STAs with the TXOP mechanism under a channel error scenario, $K_{i}=$ $[1,1,1,7]$, and SNR $=13 \mathrm{~dB}$.

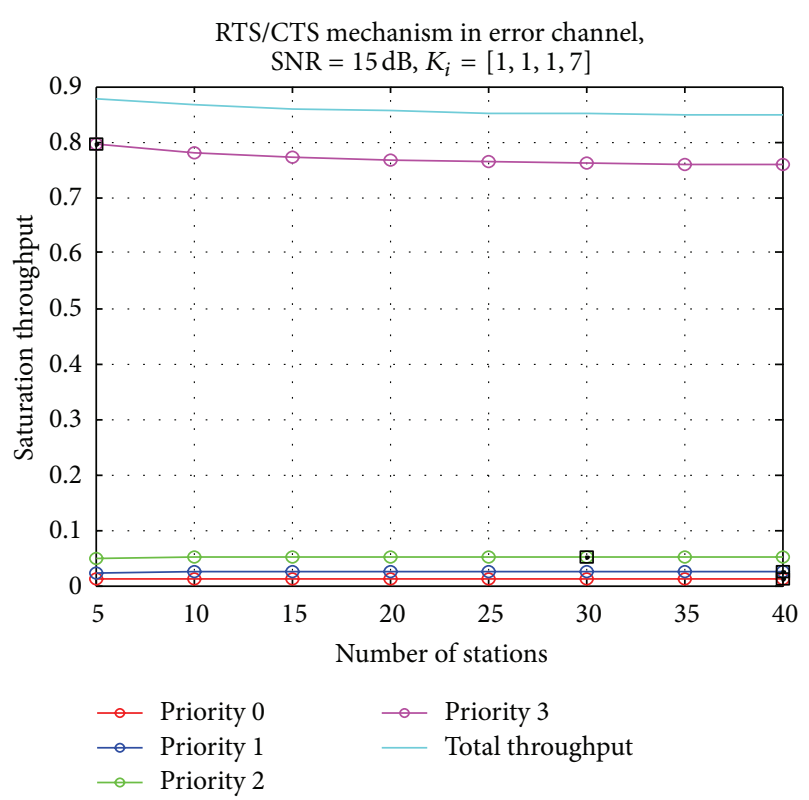

FIGURE 8: Saturation throughput of the different priority STAs with the TXOP mechanism under a channel error scenario, $K_{i}=$ $[1,1,1,7]$, and $\mathrm{SNR}=15 \mathrm{~dB}$.

The STA chooses a backoff delay of the next stage $j$ after an unsuccessful transmission at stage $j-1$ in the priority $i$ class:

$$
P\{i, j, k \mid i, j-1,0\}=\frac{p_{i}}{W_{i, j}}, \quad 1 \leq j \leq m, 0 \leq k \leq W_{i, j}-1 .
$$

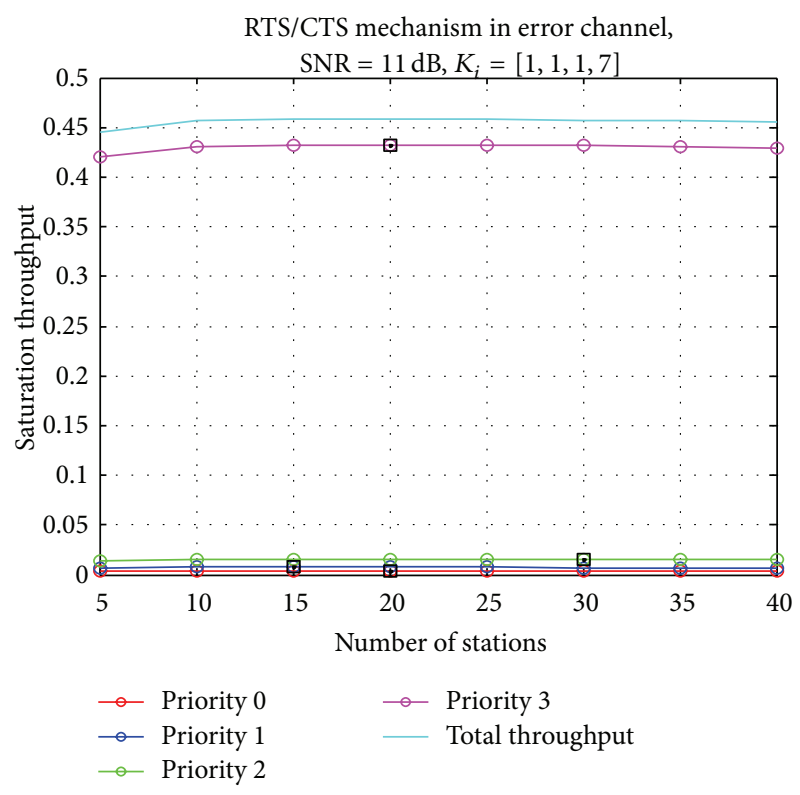

FIGURE 9: Saturation throughput with the TXOP mechanism by differentiating the retry limit.

Let $b_{i, j, k}=\lim P_{r}\{s(i, t)=j, b(i, t)=k\}$ be the stationary distribution of the Markov chain. We can derive the following relations by chain regularities:

$$
\begin{aligned}
b_{i,-1,0}=\left(2 p_{i}\left(1-p_{i}\right)^{2}\left(1-2 p_{i}\right)\right) \\
\times\left(2 p_{i}\left(1-p_{i}\right)^{2}\left(1-2 p_{i}\right)\right. \\
\quad+W_{i, 0}\left(1-\left(2 p_{i}\right)^{m}\right)\left(1-p_{i}\right) \\
\left.+\left(1+W_{i, 0}\left(2 p_{i}\right)^{m}\right)\left(1-2 p_{i}\right)\right)^{-1} .
\end{aligned}
$$

Let $\tau_{i}$ be the probability that a STA in the priority $i$ class transmits during a time slot $e$. We then have

$$
\tau_{i}=b_{i,-1,0}+\sum_{j=0}^{m-1} b_{i, j, 0}+b_{i, m, 0}=\frac{1}{1-p_{i}} b_{i,-1,0} .
$$

2.3. TXOP Mechanism. In IEEE 802.11, the TXOP is a time period when a particular station (STA) that wins the channel access has the right to initiate transmission along with the EDCA parameters of AIFS[AC], $C W_{\min }[\mathrm{AC}]$, and $C W_{\max }[\mathrm{AC}]$. During the TXOP, STA is allowed to transmit more than one frame with a short inter-frame (SIFS) time gap between an ACK and the subsequent frame transmission without the backoff process. Since SIFS is used for frame separations, the other STAs cannot gain channel access because they must wait for at least a DIFS interval. The TXOP frame structure is shown in Figure 2. 


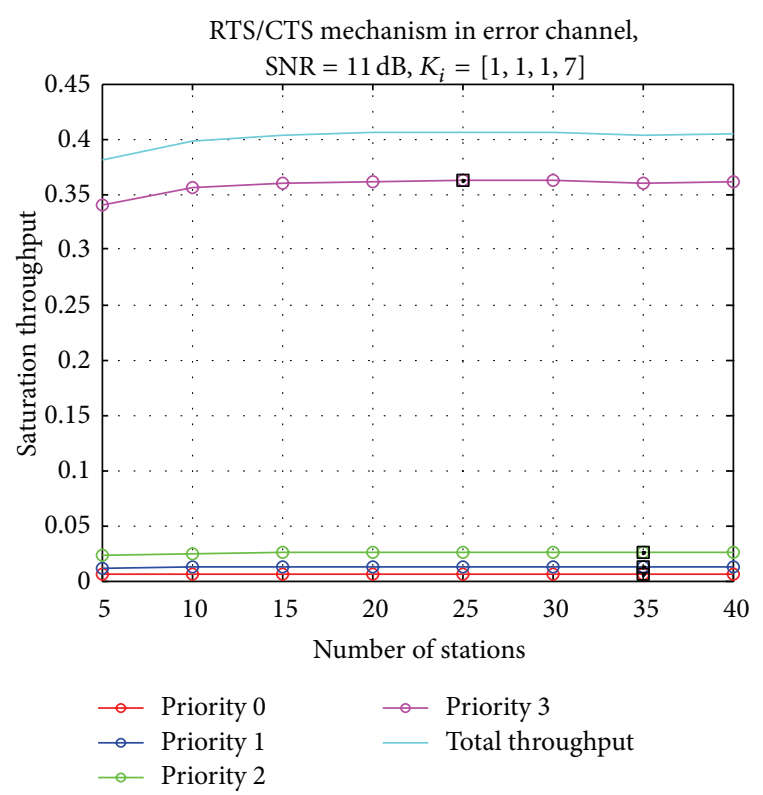

FIGURE 10: Saturation throughput with the TXOP mechanism by differentiating the retry limit.

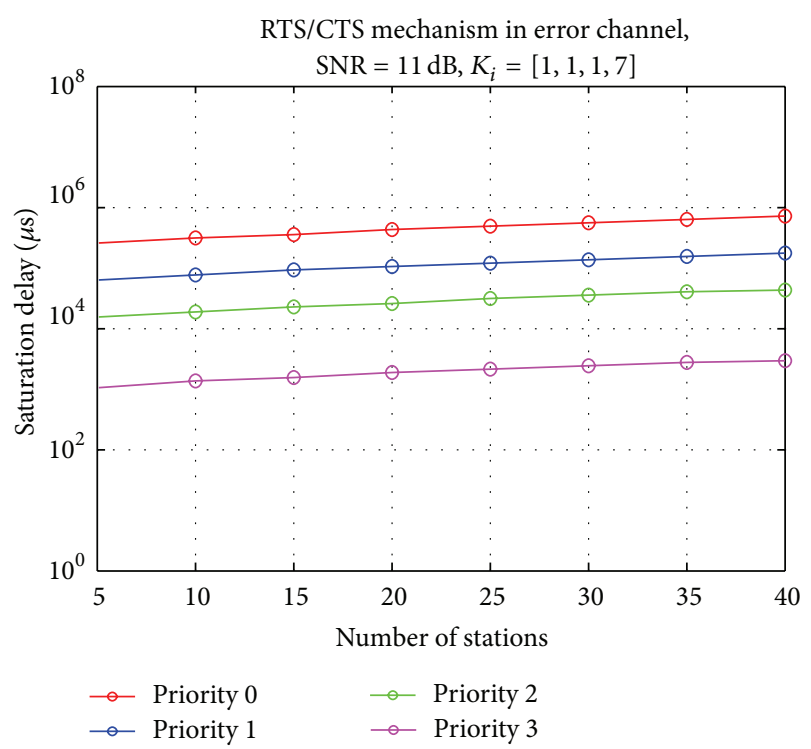

FIGURE 11: Saturation delay with the TXOP mechanism by differentiating the retry limit.

\section{Performance Analysis of EDCA with TXOP Mechanism}

The purpose of our analysis is to evaluate the saturation throughput and the delay performances of EDCA with TXOP mechanism under ideal channel and channel error scenarios. Based on the previous description, we can derive the close forms for system performance metrics of saturation throughput and delay.

From the analysis that was depicted in the previous chapter, the transmission probability is related to $C W$ size

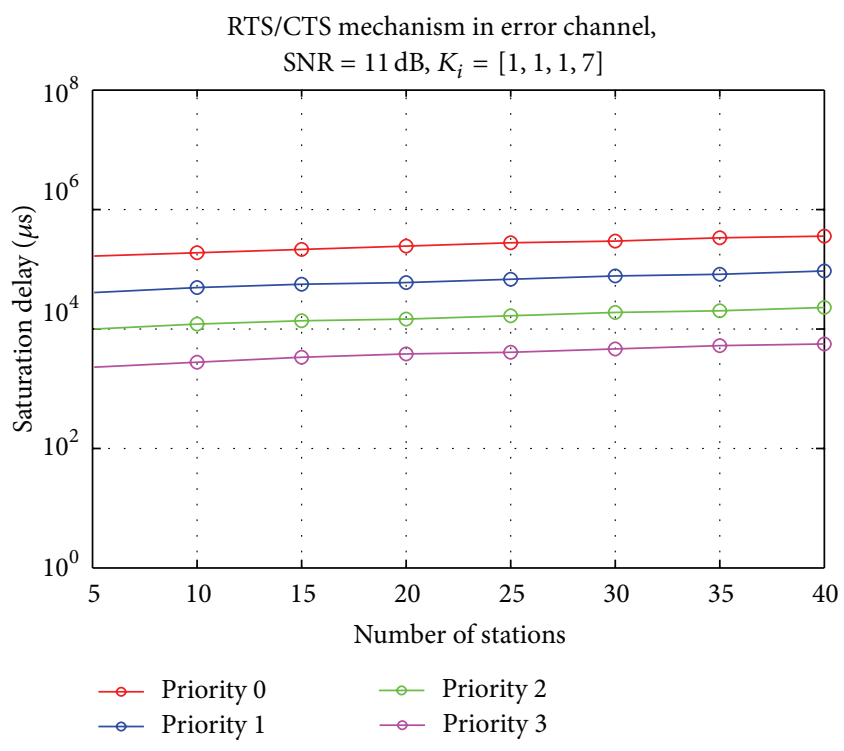

FIGURE 12: Saturation delay with the TXOP mechanism by differentiating the retry limit.

and AIFS $[\mathrm{AC}]$. When deriving the saturation throughput and delay from different TXOP durations, the collision probability and the transmission probability of an STA are the same as those derived in chapter 4 . However, the equations relating to the transmission time of each successful contention will be different for the different ACs; that is, different ACs are assigned to different TXOP durations. We assume that the system is under an environment of channel error having the RTS/CTS CSMA/CA mechanism. Thus, we can obtain

$$
\begin{gathered}
S_{i}=\left(p_{s, i} * E\left[T_{L_{i}}\right]\right) \\
\times\left(\left(1-p_{i}\right) \sigma+\sum_{i=0}^{N-1} p_{s, i}\left(1-P_{e, i}\right) T_{S, i}+\left[p_{i}-p_{s, i}\right] T_{C, i}\right. \\
+\sum_{i=0}^{N-1} p_{s, i}\left(P_{e, i}^{\mathrm{RTS}} T_{e, i}^{\mathrm{RTS}}+P_{e, i}^{\mathrm{CTS}} T_{e, i}^{\mathrm{CTS}}\right. \\
\left.\left.+P_{e, i}^{\mathrm{DATA}} T_{e, i}^{\mathrm{DATA}}+P_{e, i}^{\mathrm{ACK}} T_{e, i}^{\mathrm{ACK}}\right)\right)^{-1},
\end{gathered}
$$

$$
\begin{aligned}
E\left[T_{L_{i}}\right]= & \left\{\sum_{l=1}^{K_{i}-1} l *\left(\left(1-P_{e, i}\right)\left(1-\widehat{P}_{e, i}\right)^{l-1} \widehat{P}_{e, i}\right)\right. \\
& \left.+K_{i} *\left(1-P_{e, i}\right)\left(1-\widehat{P}_{e, i}\right)^{K_{i}-1}\right\} * T_{L_{i}}, \\
T_{S, i}= & T_{\mathrm{RTS}}+T_{\mathrm{CTS}}+2 T_{\mathrm{SIFS}} \\
& +k_{i} *\left(T_{L_{i}}+T_{\mathrm{ACK}}+2 T_{\mathrm{SIFS}}\right)+\operatorname{AIFS}(i),
\end{aligned}
$$

where $T_{S, i}$ is the TXOP duration which equals the time needed to successively transmit $k_{i}$ frames. When the system 
is under the environment of a channel error, there are three kinds of transmission error frames: (1) the RTS/CTS frames transmission error; when the RTS/CTS frames transmit an error, the STA cannot determine whether the error is caused by a collision or channel error; (2) the data frames transmission error. (3) The ACK frames transmission error. The probabilities of an unsuccessful transmission can be written as

$$
\begin{gathered}
P_{e, i}=1-\left(1-P_{b}\right)^{L_{\mathrm{RTS}}+L_{\mathrm{CTS}}+L_{i}+L_{\mathrm{ACK}},} \\
\widehat{P}_{e, i}=1-\left(1-P_{b}\right)^{L_{i}}, \\
P_{e, i}^{\mathrm{RTS}}=1-\left(1-P_{b}\right)^{L_{\mathrm{RTS}}}, \\
P_{e, i}^{\mathrm{CTS}}=\left(1-P_{b}\right)^{L_{\mathrm{RTS}}}\left(1-\left(1-P_{b}\right)^{L_{\mathrm{CTS}}}\right), \\
P_{e, i}^{\mathrm{DATA}}=\left(1-P_{b}\right)^{L_{\mathrm{RTS}}+L_{\mathrm{CTS}}}\left(1-\left(1-P_{b}\right)^{L_{i}}\right), \\
P_{e, i}^{\mathrm{ACK}}=\left(1-P_{b}\right)^{L_{\mathrm{RTS}}+L_{\mathrm{CTS}}+L_{i}}\left(1-\left(1-P_{b}\right)^{L_{\mathrm{ACK}}}\right),
\end{gathered}
$$

where $P_{b}$ is the bit error rate and $L_{x x x}$ is the length of different types of frames. $P_{e, i}^{x x x}$ is the frame-error rate of the frame type $x x x$ for the priority $i$ class. $T_{e, i}^{x x x}$ is the time duration caused by the frame errors of the different frame types $x x x$. From the description, we can get the time duration of the different frame types $x \times x$ as follows:

$$
\begin{gathered}
T_{e, i}^{\mathrm{RTS}}=T_{\mathrm{RTS}}+T_{\mathrm{CTS}}^{\mathrm{timeout}}+\operatorname{AIFS}[i], \\
T_{e, i}^{\mathrm{CTS}}=T_{\mathrm{RTS}}+T_{\mathrm{SIFS}}+\operatorname{AIFS}[i], \\
T_{e, i}^{\mathrm{DATA}}=T_{\mathrm{RTS}}+T_{\mathrm{CTS}}+\operatorname{AIFS}[i] \\
+2 T_{\mathrm{SIFS}}+T_{H}+T_{L_{i}}+T_{\mathrm{ACK}}^{\text {timeout }}, \\
T_{e, i}^{\mathrm{ACK}}=T_{\mathrm{RTS}}+T_{\mathrm{CTS}}+\operatorname{AIFS}[i]+3 T_{\mathrm{SIFS}}+T_{H}+T_{L_{i}} .
\end{gathered}
$$

From the previous description, the saturation delay in a channel error scenario for the priority $i$ class can be defined as

$$
\begin{aligned}
E\left[D_{i}\right]= & E\left[X_{i}\right] \sigma+E\left[B_{i}\right]\left(\left(1-p_{e, i}\right) p_{s, i} T_{S, i}+\left(p_{i}-p_{S, i}\right) T_{C, i}\right) \\
& +E\left(N_{i, m}\right)\left(T_{C, i}+T_{O}\right)+\left(1-p_{e, i}\right) T_{S, i}
\end{aligned}
$$

where the first term on the right hand side of (15) is the time for the backoff process, the second term is the time of the frozen backoff counter, the third term is the time needed to deal with collisions, and the fourth term is the successful transmission time, where $E\left[N_{i, \text { retry }}\right]$ denotes the average number of retries of a frame for the priority $i$ until it is successfully received and depicted as follows:

$$
E\left(N_{i, \text { retry }}\right)=\sum_{j=0}^{m_{i, \text { retry }}} \frac{j p_{i}^{j}\left(1-p_{i}\right)\left(1-P_{e, i}\right)}{1-p^{m_{i} \text {,retry+1 }}},
$$

$$
\begin{gathered}
E\left(X_{i}\right)=\sum_{j=0}^{m_{i, \text { retry }}} \frac{p_{i}^{j}\left(1-p_{i}\right)\left(1-P_{e, i}\right)}{1-p^{m_{i, \text { retry }}+1}} \sum_{h=0}^{j} \frac{W_{i, h}-1}{2}, \\
E\left(B_{i}\right)=\frac{E\left(X_{i}\right)}{\left(1-p_{i}\right)} p_{i} .
\end{gathered}
$$

We denote that $E\left(X_{i}\right)$ and $E\left(B_{i}\right)$ are the total number of idle and busy slots that the frame encounters, respectively, during the backoff process for the priority $i$ class.

\section{Performance Evaluation}

Our simulation model is built on Dedicated Short Range Communications (DSRC). DSRC is a block of spectra in the 5.850 to $5.925 \mathrm{GHz}$ band. It is a short-to-medium range communications service that supports both public safety and private operations in roadside-to-vehicle and vehicle-tovehicle communication environments. DSRC complements cellular communications by providing very high data transfer rates while minimizing latency in the link of short range [13]. DSRC is a part of Intelligent Transportation Systems (ITS). Compared with the existing wireless communication techniques of ITS, such as wireless AM and FM broadcasters, Global System for Mobile Communications (GSM), Global Positioning System (GPS), satellite telephone service in the $2.3 \mathrm{GHz}$ band, and collision avoidance radar system in the $77 \mathrm{GHz}$ band, DSRC can support low latency $(<50 \mathrm{~ms})$ and very high data transfer rates. Some possible applications in public safety are intersection collision avoidance, transit or emergency vehicle signal priority, vehicle safety inspection, and approaching emergency vehicle warning. Other possible applications in private operations are in-vehicle signing, electronic parking payments, gas station payments, and internet access services [14]. The simulation parameters are as follows: Frame payload $=1023$ bytes, MAC header $=34$ bytes, $\mathrm{PHY}$ header $=16$ bytes, $\mathrm{ACK}=14$ bytes, $\mathrm{RTS}=20$ bytes, CTS $=14$ bytes, SIFS $=20 \mu \mathrm{s}$, DIFS $=50 \mu \mathrm{s}$, propagation delay $=1 \mu \mathrm{s}$, and slot time $=20 \mu \mathrm{s}$. First, we finished the simulation of the TXOP mechanism with the RTS/CTS CSMA/CA access scheme under the channel error scenarios and compared it with the model that is without the TXOP mechanism. Figure 3 shows the saturation throughput performance of the TXOP mechanism with the RTS/CTS CSMA/CA access scheme for different priority STAs under a channel error scenario. Figure 4 depicts the saturation throughput performance of different priority STAs using the RTS/CTS CSMA/CA access scheme without the TXOP mechanism under a channel error scenario. From the results, we can conclude that the model with the TXOP mechanism under a channel error scenario can achieve higher performance than the model that is without the TXOP mechanism because the model with the TXOP mechanism is allowed to transmit more than one frame with a short interframe (SIFS) time gap between an ACK and the subsequent frame transmission without the backoff process.

The throughput performances with the TXOP mechanism that considers different SNR conditions are shown in 
Figures 5, 6, 7, and 8. From the results, we can conclude that the TXOP mechanism with a higher SNR can achieve a higher performance. The saturation delay performances of different priority STAs using the RTS/CTS CSMA/CA scheme with the TXOP mechanism under a nonideal channel scenario are shown in Figures 9, 10, 11, and 12. In the lower SNR scenario due to the channel error increase, the saturation delay increases. Figures 9, 10, 11, and 12 have the following parameters: the retry limit of priority 3 is changed from 3 to 4 ; the lower the retry limit is, the higher the saturation throughput of priority 3 is, while the saturation throughput of the other priority classes decreases and their delay increases, as the priority 3 has a lower retry limit.

\section{Conclusion}

In IEEE 1609 WAVE, it supports a variety of safety and nonsafety applications with eight levels of access category supports QoS using EDCA in IEEE 802.11. In this paper, we considered the Markov chain model of IEEE 802.11 EDCA under a non-ideal channel. In the non-ideal channel, the frame may be received unsuccessfully due to a channel error. When the frame is received unsuccessfully, it results in an increase in the saturation delay and a reduction in the saturation throughput. In order to improve the efficiency, we used the TXOP mechanism with the proposed model in a non-ideal channel scenario. The proposed model with the TXOP mechanism under a channel error scenario can achieve a higher performance than that without the TXOP mechanism due to fact that the modified model with the TXOP mechanism is allowed to transmit multiple frames during the TXOP duration.

\section{References}

[1] Y. Xiao, "QoS guarantee and provisioning at the contentionbased wireless MAC layer in the IEEE 802.11 wireless LANs," IEEE Wireless Communications Magazine, vol. 13, pp. 14-21, 2006.

[2] D. Gu and J. Zhang, "QoS enhancement in IEEE802.11 wireless local area networks," IEEE Communications Magazine, vol. 41, no. 6, pp. 120-124, 2003.

[3] S. Choi, J. del Prado, S. Shankar N, and S. Mangold, "IEEE 802.11e Contention-based channel access (EDCF) performance evaluation," in Proceedings of the International Conference on Communications (ICC '03), pp. 1151-1156, May 2003.

[4] J. Deng and R. S. Chang, "A priority scheme for IEEE 802.11 DGF access method," IEICE Transactions on Communications, vol. 82, no. 1, pp. 96-102, 1999.

[5] I. Aad and C. Castelluccia, "Differentiation mechanisms for IEEE 802.11," in Proceedings of the 20th Annual Joint Conference on the IEEE Computer and Communications Societies (IEEE INFOCOM '01), pp. 209-218, April 2001.

[6] A. Veres, A. T. Campbell, M. Barry, and L. H. Sun, "Supporting service differentiation in wireless packet networks using distributed control," IEEE Journal on Selected Areas in Communications, vol. 19, no. 10, pp. 2081-2093, 2001.

[7] S. Gräfling, P. Mähönen, and J. Riihijärvi, "Performance evaluation of IEEE, 1609 WAVE and IEEE 802. 11p for vehicular communications," in Proceedings of the International Conference on Ubiquitous and Future Networks (ICUFN '10), pp. 344-348, Jeju Island, Republic of Korea, June 2010.

[8] J. R. Gallardo, D. Makrakis, and H. T. Mouftah, "Performance analysis of the EDCA medium access mechanism over the control channel of an IEEE 802.11p WAVE vehicular network," in Proceedings of the IEEE International Conference on Communications (ICC '09), pp. 1-6, Dresden, Germany, June 2009.

[9] S. W. Chang, J. Cha, and S. S. Sun, "Adaptive EDCA mechanism for vehicular ad-hoc network," in Proceedings of the International Conference on Information Networking (ICOIN '12), pp. 379-383, Bali, Indonesia, February 2012.

[10] IEEE 802.11 WG, Part 11: Wireless LAN Medium Access Control (MAC) and Physical Layer (PHY) Specification, 1999.

[11] G. Bianchi, "Performance analysis of the IEEE 802.11 distributed coordination function," IEEE Journal on Selected Areas in Communications, vol. 18, no. 3, pp. 535-547, 2000.

[12] Y. Xiao and J. Rosdahl, "Throughput and delay limits of IEEE 802. 11," IEEE Communications Letters, vol. 6, no. 8, pp. 355-357, 2002.

[13] J. Zhu and S. Roy, "MAC for dedicated short range communications in intelligent transport system," IEEE Communications Magazine, vol. 41, no. 12, pp. 60-67, 2003.

[14] http://en.wikipedia.org/wiki/DSRC. 

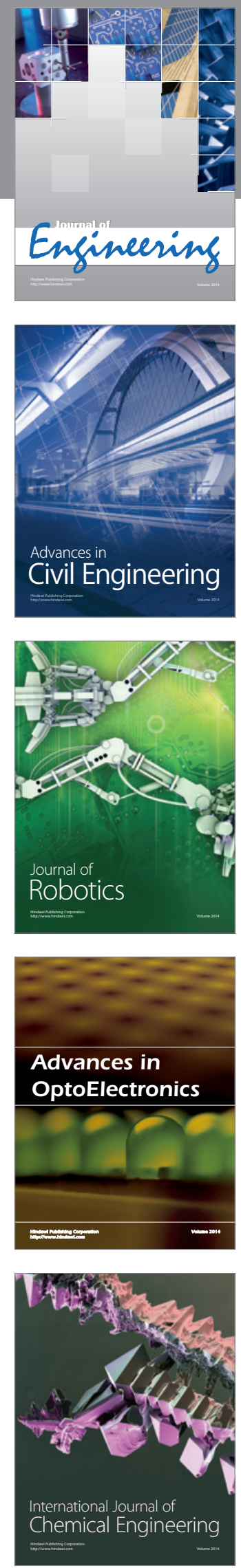

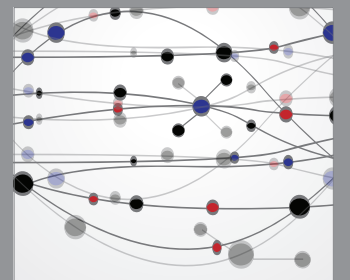

The Scientific World Journal
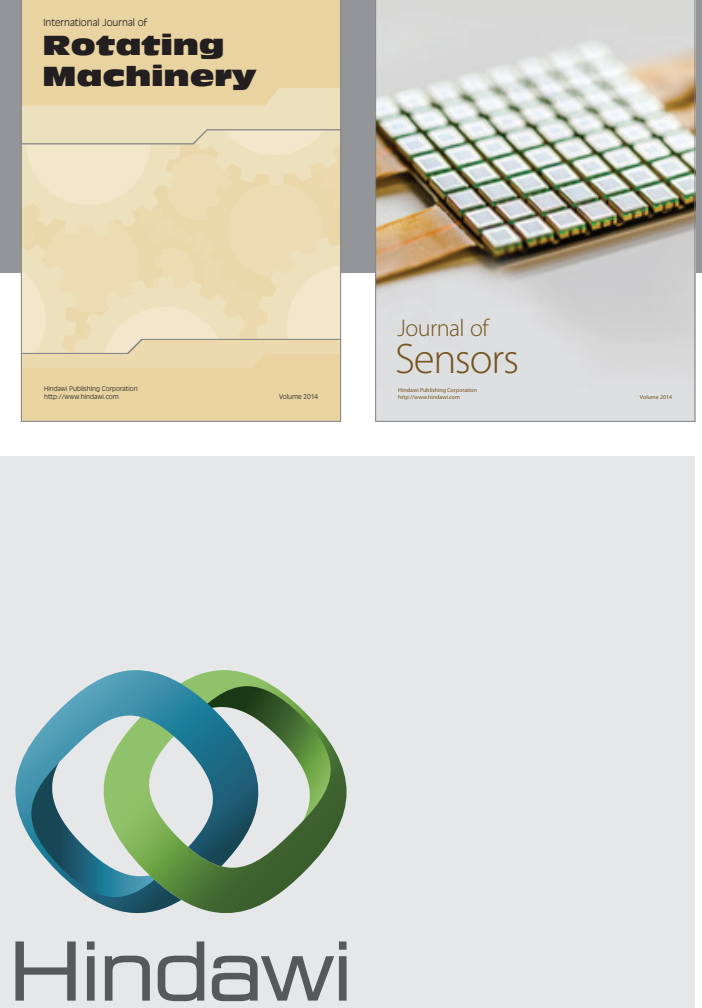

Submit your manuscripts at http://www.hindawi.com
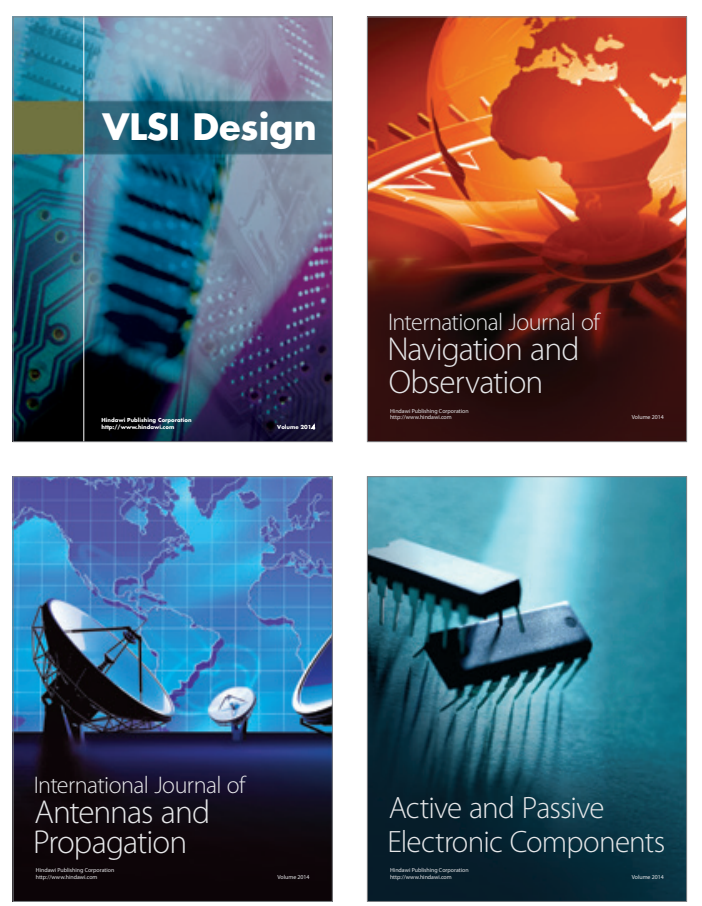
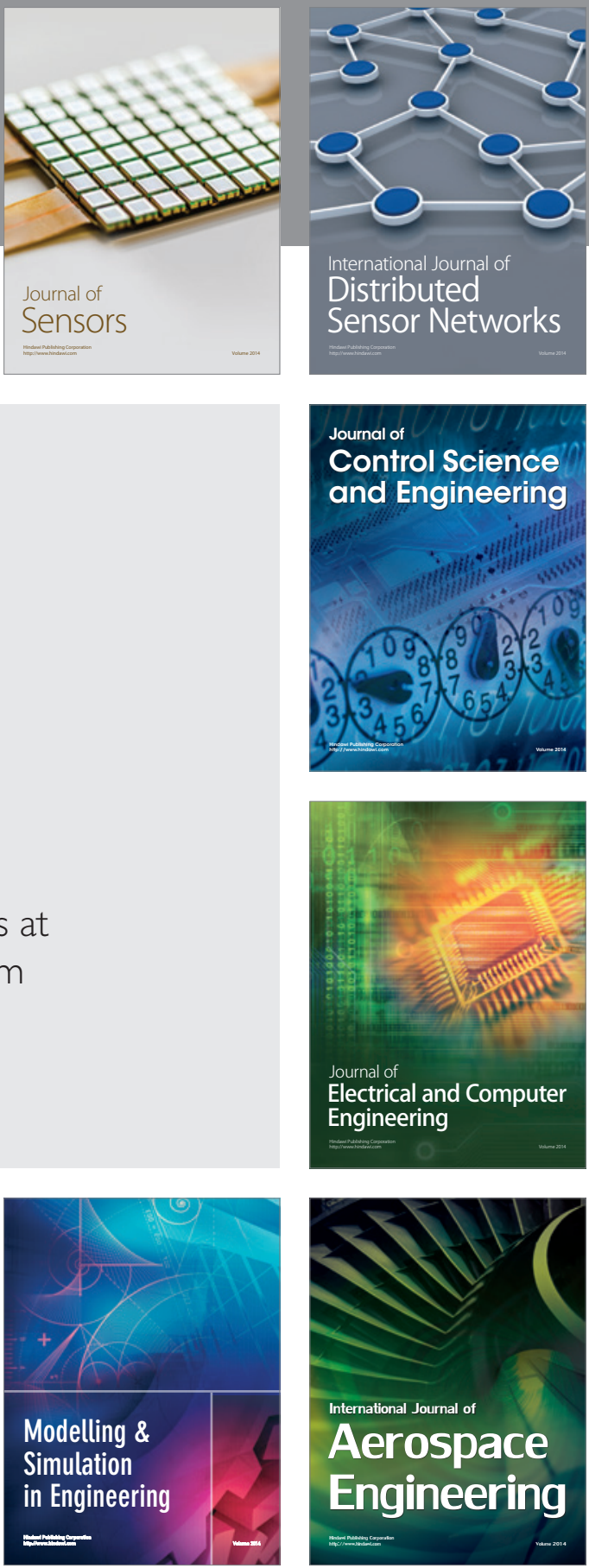

Journal of

Control Science

and Engineering
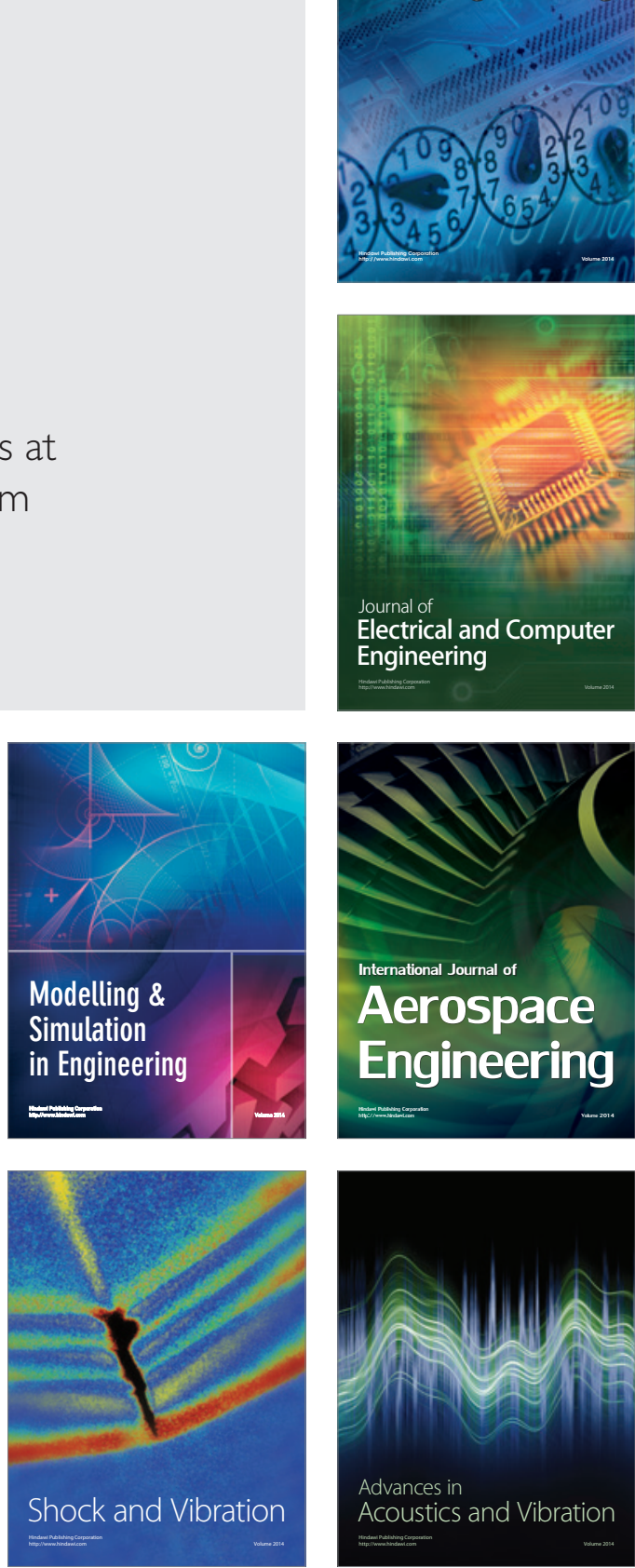